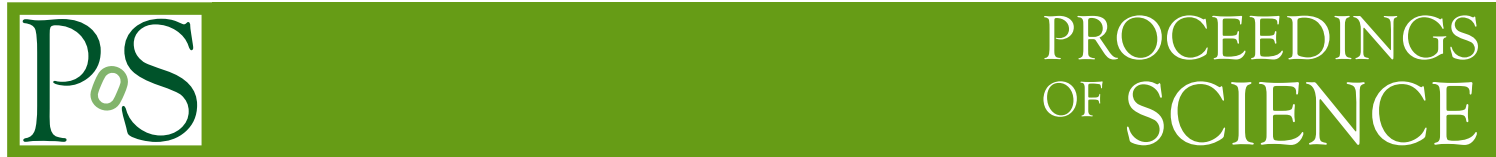

\title{
Beam energy dependence of the anisotropic flow coefficients $\mathbf{v}_{n}$
}

\author{
Niseem Magdy* (For the STAR Collaboration) \\ Department of Chemistry, Stony Brook University, Stony Brook, NY, 11794-3400, USA \\ E-mail: niseemm@gmail.com
}

Recent STAR measurements of the anisotropic flow coefficients, $\mathrm{v}_{n}$, are presented for $\mathrm{Au}+\mathrm{Au}$ collisions spanning the beam energy range $\sqrt{s_{N N}}=7.7-200 \mathrm{GeV}$. The measurements indicate dependences on harmonic number, $n$, transverse momentum $\left(p_{T}\right)$, pseudorapidity $(\eta)$, collision centrality (cent) and beam energy $\left(\sqrt{s_{N N}}\right)$ which could serve as important constraints to test different initial-state models and to aid precision extraction of the temperature dependence of the specific shear viscosity.

Critical Point and Onset of Deconfinement

, 7-11 August 2017

The Wang Center, Stony Brook University, Stony Brook, NY

${ }^{*}$ Speaker. 


\section{Introduction}

A major goal of the heavy-ion experimental program at the Relativistic Heavy Ion Collider (RHIC) is to study the properties of the strongly interacting quark-gluon plasma (QGP) created in ion-ion collisions. Recently, many studies have emphasized the use of anisotropic flow measurements to study the transport properties of the QGP [1-7]. An important question in many of these studies has been the role of initial-state fluctuations and their influence on the uncertainties associated with the extraction of $\eta / s$ for the QGP [8,9]. This work presents new measurements for the anisotropic flow coefficients, $\mathrm{v}_{n>1}$, and the rapidity-even dipolar flow coefficient, $\mathrm{v}_{1}^{\text {even }}$, with an eye toward developing new constraints which could aid a distinction between different initial-state models and hence, facilitate a more precise extraction of the specific shear viscosity $\eta / s[10,11]$.

Anisotropic flow is characterized by the Fourier coefficients, $\mathrm{v}_{n}$, obtained from a Fourier expansion of the azimuthal angle $(\phi)$ distribution of the particles emitted in the collisions [12]:

$$
\frac{d N}{d \phi} \propto 1+2 \sum_{n=1} \mathrm{v}_{\mathrm{n}} \cos \left(n\left(\phi-\Psi_{n}\right)\right),
$$

where $\Psi_{n}$ represents the azimuthal angle of the $n^{\text {th }}$-order event plane; the coefficients, $\mathrm{v}_{1}, \mathrm{v}_{2}$ and $\mathrm{v}_{3}$ are commonly called directed, elliptic, and triangular flow, respectively. The flow coefficients, $\mathrm{v}_{n}$, are related to the two-particle Fourier coefficients, $\mathrm{v}_{n, n}$, as:

$$
\mathrm{v}_{\mathrm{n}, \mathrm{n}}\left(p_{\mathrm{T}}^{a}, p_{\mathrm{T}}^{b}\right)=\mathrm{v}_{\mathrm{n}}\left(p_{\mathrm{T}}^{a}\right) \mathrm{v}_{\mathrm{n}}\left(p_{\mathrm{T}}^{b}\right)+\delta_{\mathrm{NF}}
$$

where a and $\mathrm{b}$ are particles selected with $p_{\mathrm{T}}^{a}$ and $p_{\mathrm{T}}^{b}$ respectively, and $\delta_{\mathrm{NF}}$ is a so-called nonflow (NF) term, which includes possible short-range contributions from resonance decays, BoseEinstein correlations and near-side jets, and long-range contributions from the global momentum conservation (GMC) [13-15]. The short-range contributions can be reduced by employing a pseudorapidity gap, $\Delta \eta$. However, the effects of GMC must be explicitly considered. For the current analysis, a simultaneous fitting procedure, outlined below, was used to account for GMC.

\section{Measurements}

The correlation function technique was used to measure the two-particle $\Delta \phi$ correlations:

$$
C_{r}(\Delta \phi, \Delta \eta)=\frac{(d N / d \Delta \phi)_{\text {same }}}{(d N / d \Delta \phi)_{\text {mixed }}},
$$

where $(d N / d \Delta \phi)_{\text {same }}$ represent the normalized azimuthal distribution of particle pairs from the same event and $(d N / d \Delta \phi)_{\text {mixed }}$ represents the normalized azimuthal distribution for particle pairs in which each member is selected from a different event but with a similar classification for the collision vertex location, centrality, etc. The pseudorapidity requirement $|\Delta \eta|>0.7$ was also imposed on track pairs to minimize non-flow contributions associated with the short-range correlations.

The two-particle Fourier coefficients, $\mathrm{v}_{n, n}$, are obtained from the correlation function as:

$$
\mathrm{v}_{\mathrm{n}, \mathrm{n}}=\frac{\sum_{\Delta \phi} C_{r}(\Delta \phi, \Delta \eta) \cos (n \Delta \phi)}{\sum_{\Delta \phi} C_{r}(\Delta \phi, \Delta \eta)}
$$


and then used to extract $\mathrm{v}_{1}^{\text {even }}$ via a simultaneous fit of $\mathrm{v}_{1,1}$ as a function of $p_{T}^{\mathrm{b}}$, for several selections of $p_{T}^{a}$ with Eq. 1.2:

$$
\mathrm{v}_{1,1}\left(p_{\mathrm{T}}^{a}, p_{\mathrm{T}}^{b}\right)=\mathrm{v}_{1}^{\text {even }}\left(p_{\mathrm{T}}^{a}\right) \mathrm{v}_{1}^{\mathrm{even}}\left(p_{\mathrm{T}}^{b}\right)-C p_{\mathrm{T}}^{a} p_{\mathrm{T}}^{b}
$$

Here, $C \propto 1 /\left(\langle\right.$ Mult $\left.\rangle\left\langle p_{T}^{2}\right\rangle\right)$ takes into account the non-flow correlations induced by a global momentum conservation $[15,16]$ and $\langle$ Mult $\rangle$ is the corrected mean multiplicity. For a given centrality selection, the left hand side of Eq. 2.3 represents the $N \times N$ matrix which we fit with the right hand side using $N+1$ parameters; $\mathrm{N}$ values of $\mathrm{v}_{1}^{\text {even }}\left(p_{\mathrm{T}}\right)$ and one additional parameter $C$, accounting for the momentum conservation [17].
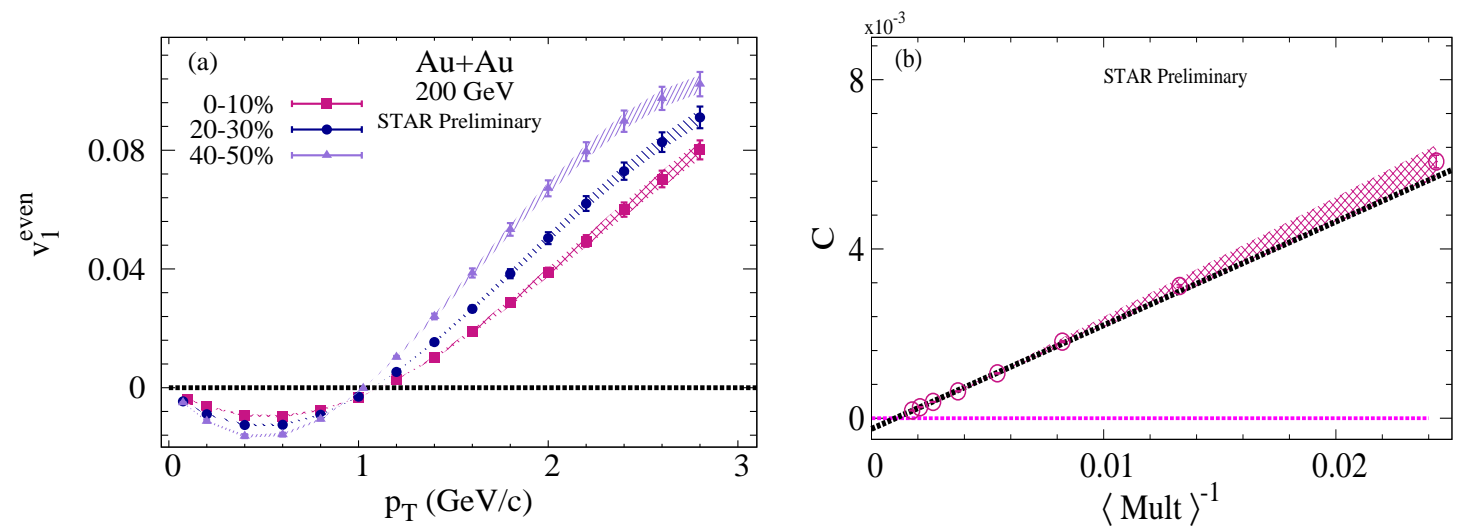

Figure 1: (a) The extracted values of $v_{1}^{e v e n}$ vs. $p_{T}$ for $\mathrm{Au}+\mathrm{Au}$ collisions at $\sqrt{s_{N N}}=200 \mathrm{GeV}$. (b) $\mathrm{A}$ representative set of the associated values of $C$ vs. $\langle M u l t\rangle^{-1}$ from the same fits. The shaded bands represent the systematic uncertainty.

\section{Results}

Representative results for $\mathrm{v}_{1}^{\text {even }}$ and $\mathrm{v}_{n \geq 2}$ for $\mathrm{Au}+\mathrm{Au}$ collisions at several different collision energies are summarized in Figs. 1, 2, 3, 4 and 5.

The values of $v_{1}^{\text {even }}\left(p_{\mathrm{T}}\right)$ extracted for different centrality selections $(0-10 \%, 20-30 \%$ and $40-$ $50 \%)$ are shown in Fig. 1(a). They indicate the characteristic pattern of a change from negative $\mathrm{v}_{1}^{\text {even }}\left(p_{\mathrm{T}}\right)$ at low $p_{\mathrm{T}}$ to positive $\mathrm{v}_{1}^{\text {even }}\left(p_{\mathrm{T}}\right)$ for $p_{T}>1 \mathrm{GeV} / \mathrm{c}$. They also show the expected increase of $\mathrm{v}_{1}^{\text {even }}$ as collisions become more peripheral, in line with the expected centrality dependence of the dipole asymmetry $\varepsilon_{1}$, where $\varepsilon_{1} \equiv\left|\left(r^{3} e^{i \phi}\right)\right| / r^{3}[18,19]$. Fig. 1(b) shows the results for the associated momentum conservation coefficients, $C$; they indicate the expected linear dependence on $\langle\text { Mult }\rangle^{-1}$.

Figure 2 and 3 show $p_{T}$ and $\eta$ differential $\mathrm{v}_{\mathrm{n} \geq 2}$ measurements for the centrality selection $0-40 \%$, for a representative set of beam energies. Fig. 2 indicates a sizable dependence of the magnitude of $\mathrm{v}_{n}$ on $p_{T}$ and the harmonic number, $n$, with similar trends for each beam energy. Figure 3 shows a similarly strong $n$ dependence for $\mathrm{v}_{\mathrm{n} \geq 2}$ but with a much weaker $\eta$ dependence.

The centrality dependence of $\mathrm{v}_{n \geq 2}$ is shown in Fig. 4 for the same representative set of beam energies. They indicate a weak centrality dependence for the higher harmonics, which all decrease 


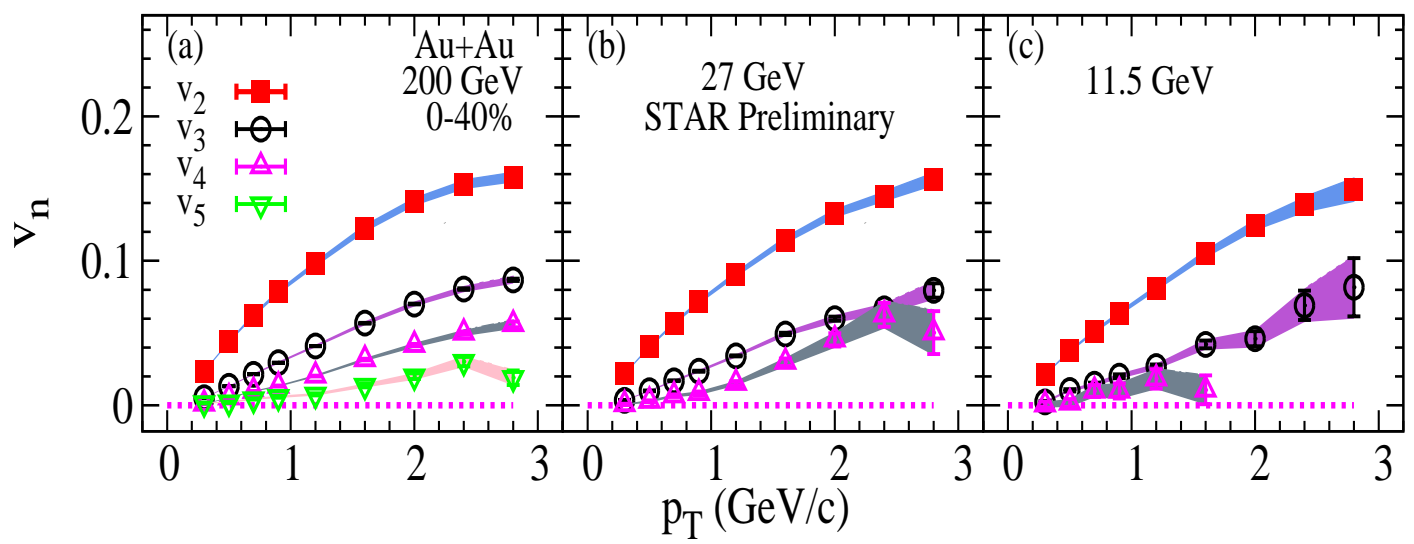

Figure 2: Examples of $\mathrm{v}_{n}\left(p_{T}\right)$ as a function of $p_{T}$ for charged particles in $0-40 \%$ central $\mathrm{Au}+\mathrm{Au}$ collisions. The shaded bands represent the systematic uncertainty.

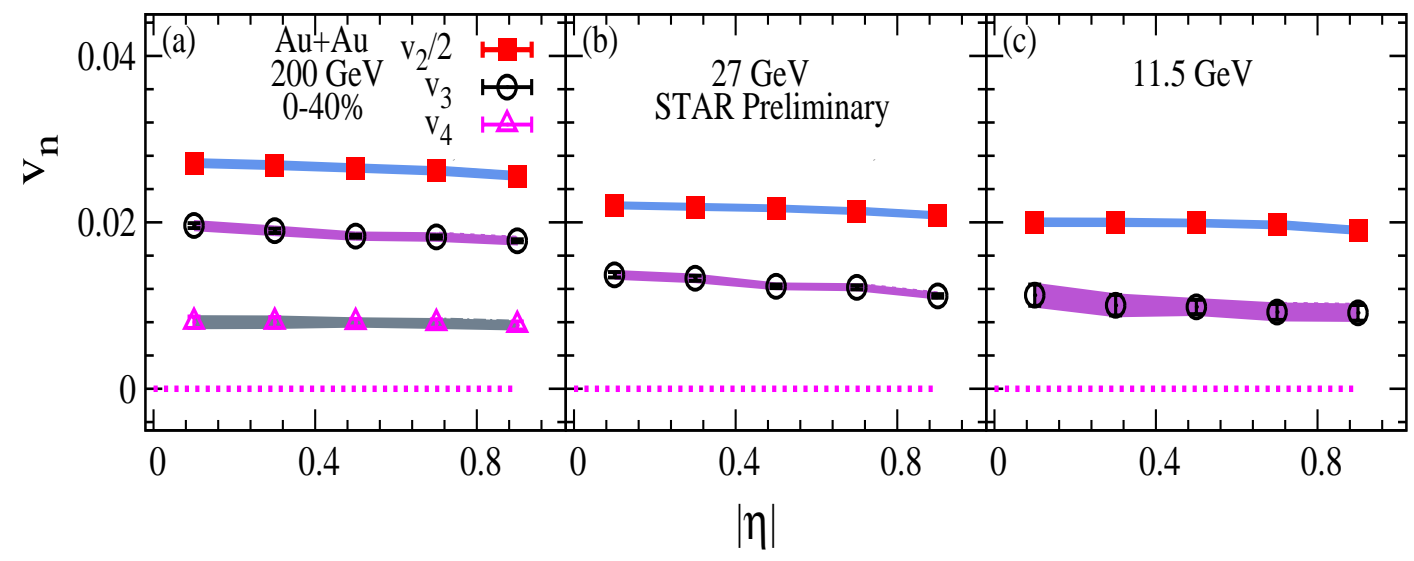

Figure 3: Examples of $\mathrm{v}_{n}(|\eta|)$ as a function of $|\eta|$ for charged particles in 0-40\% central Au+Au collisions. The shaded bands represent the systematic uncertainty.

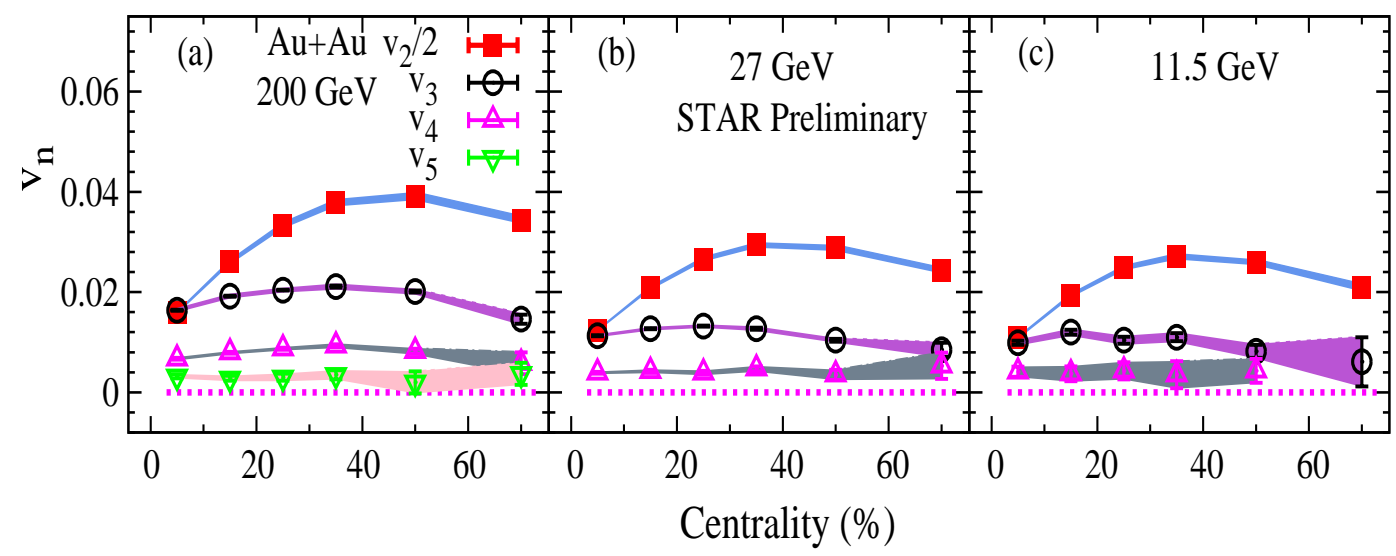

Figure 4: Examples of $\mathrm{v}_{n}($ Centrality\%) as a function of $\mathrm{Au}+\mathrm{Au}$ collision centrality for charged particles with $0.2<p_{T}<4 \mathrm{GeV} / \mathrm{c}$. The shaded bands represent the systematic uncertainty. 


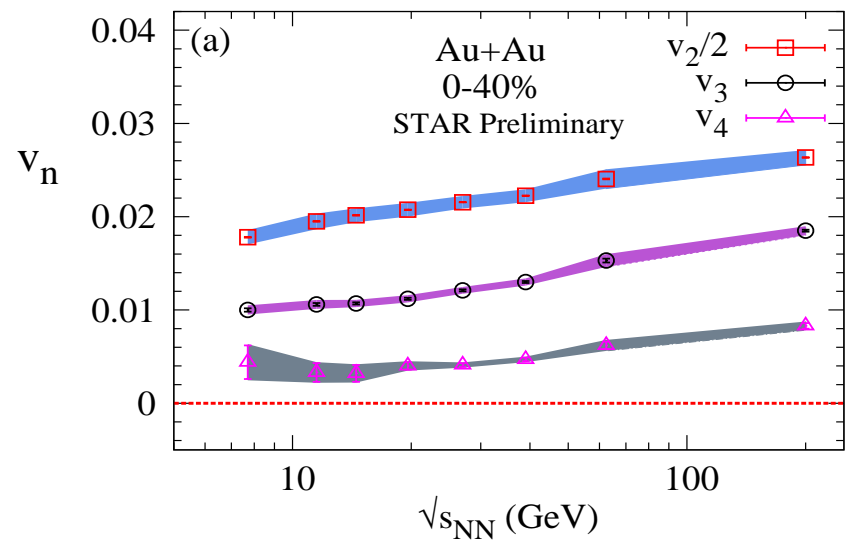

Figure 5: Examples of $\mathrm{v}_{n}\left(\sqrt{s_{\mathrm{NN}}}\right)$ for charged particles with $0.2<p_{T}<4 \mathrm{GeV} / \mathrm{c}$ and $0-40 \%$ central Au+Au collisions. The shaded bands represent the systematic uncertainty.

with decreasing values of $\sqrt{s_{\mathrm{NN}}}$. These patterns may be related to the detailed dependence of the viscous effects in the created medium, which serve to attenuate the magnitude of $v_{n}$.

Figure 5 shows the excitation functions for the $p_{T}$-integrated $v_{2,3,4}$ for $0-40 \%$ central $\mathrm{Au}+\mathrm{Au}$ collisions. They indicate an essentially monotonic trend for $\mathrm{v}_{2}, \mathrm{v}_{3}$ and $\mathrm{v}_{4}$ with $\sqrt{s_{\mathrm{NN}}}$, as might be expected for a temperature increase with $\sqrt{s_{\mathrm{NN}}}$.

\section{Conclusion}

In summary, we have performed a comprehensive set of STAR anisotropic flow measurements for Au+Au collisions at $\sqrt{s_{\mathrm{NN}}}=7.7-200 \mathrm{GeV}$. The measurements use the two-particle correlation method to extract the Fourier coefficients, $\mathrm{v}_{n>1}$, and the rapidity-even dipolar flow coefficient, $\mathrm{v}_{1}^{\text {even }}$. The rapidity-even dipolar flow measurements indicate the characteristic patterns of an evolution from negative $\mathrm{v}_{1}^{\text {even }}\left(p_{\mathrm{T}}\right)$ for $p_{\mathrm{T}}<1 \mathrm{GeV} / \mathrm{c}$ to positive $\mathrm{v}_{1}^{\text {even }}\left(p_{\mathrm{T}}\right)$ for $p_{\mathrm{T}}>1 \mathrm{GeV} / \mathrm{c}$, expected when initial-state geometric fluctuations act along with the hydrodynamic-like expansion to generate rapidity-even dipolar flow. The $\mathrm{v}_{n>1}$ measurements indicate a rich set of dependences on harmonic number $n, p_{T},|\eta|$ and centrality for versus the beam energy. These new measurements may provide additional constraints to test different initial-state models, and to aid precision extraction of the temperature dependence of the specific shear viscosity.

\section{Acknowledgments}

This research is supported by the US Department of Energy under contract DE-FG02-87ER40331.

\section{References}

[1] D. Teaney, The Effects of viscosity on spectra, elliptic flow, and HBT radii, Phys.Rev. C68 (2003) 034913, [nucl-th/0301099].

[2] R. A. Lacey and A. Taranenko, What do elliptic flow measurements tell us about the matter created in the little bang at RHIC?, PoS CFRNC2006 (2006) 021, [nucl-ex/ 0610029 ]. 
[3] B. Schenke, S. Jeon and C. Gale, Anisotropic flow in $\sqrt{s}=2.76 \mathrm{TeV} \mathrm{Pb}+\mathrm{Pb}$ collisions at the LHC, Phys.Lett. B702 (2011) 59-63, [1102 . 0575].

[4] H. Song, S. A. Bass and U. Heinz, Elliptic flow in $200 \mathrm{~A} \mathrm{GeV} \mathrm{Au+Au} \mathrm{collisions} \mathrm{and} 2.76 \mathrm{~A} \mathrm{TeV}$ $P b+P b$ collisions: insights from viscous hydrodynamics + hadron cascade hybrid model, Phys. Rev. C83 (2011) 054912, [1103.2380].

[5] H. Niemi, G. Denicol, P. Huovinen, E. Molnar and D. Rischke, Influence of a temperature-dependent shear viscosity on the azimuthal asymmetries of transverse momentum spectra in ultrarelativistic heavy-ion collisions, Phys.Rev. C86 (2012) 014909, [1203.2452].

[6] G.-Y. Qin, H. Petersen, S. A. Bass and B. Muller, Translation of collision geometry fluctuations into momentum anisotropies in relativistic heavy-ion collisions, Phys.Rev. C82 (2010) 064903, [1009.1847].

[7] STAR collaboration, N. Magdy, Viscous Damping of Anisotropic Flow in 7.7-200 GeV Au+Au Collisions, J. Phys. Conf. Ser. 779 (2017) 012060.

[8] B. Alver and G. Roland, Collision geometry fluctuations and triangular flow in heavy-ion collisions, Phys. Rev. C81 (2010) 054905, [1003.0194].

[9] R. A. Lacey, D. Reynolds, A. Taranenko, N. N. Ajitanand, J. M. Alexander, F.-H. Liu et al., Acoustic scaling of anisotropic flow in shape-engineered events: implications for extraction of the specific shear viscosity of the quark gluon plasma, J. Phys. G43 (2016) 10LT01, [1311 . 1728].

[10] J. Auvinen, I. Karpenko, J. E. Bernhard and S. A. Bass, Investigating the collision energy dependence of $\eta / s$ in RHIC beam energy scan using Bayesian statistics, 1706.03666.

[11] J. Auvinen, I. Karpenko, J. E. Bernhard and S. A. Bass, Revealing the collision energy dependence of $\eta / s$ in RHIC-BES Au+Au collisions using Bayesian statistics, Nucl. Phys. A967 (2017) 784-787, [1704.04643].

[12] A. M. Poskanzer and S. A. Voloshin, Methods for analyzing anisotropic flow in relativistic nuclear collisions, Phys. Rev. C58 (1998) 1671-1678, [nucl-ex/9805001].

[13] R. A. Lacey, The Role of elliptic flow correlations in the discovery of the sQGP at RHIC, Nucl. Phys. A774 (2006) 199-214, [nucl-ex/ 0510029 ].

[14] N. Borghini, P. M. Dinh and J.-Y. Ollitrault, Are flow measurements at SPS reliable?, Phys. Rev. C62 (2000) 034902, [nucl-th/0004026].

[15] ATLAS collaboration, G. Aad et al., Measurement of the azimuthal anisotropy for charged particle production in $\sqrt{s_{N N}}=2.76 \mathrm{TeV}$ lead-lead collisions with the ATLAS detector, Phys. Rev. C86 (2012) 014907, [1203.3087].

[16] E. Retinskaya, M. Luzum and J.-Y. Ollitrault, Directed flow at midrapidity in $\sqrt{s_{N N}}=2.76 \mathrm{TeV}$ Pb+Pb collisions, Phys. Rev. Lett. 108 (2012) 252302, [1203. 0931$].$

[17] J. Jia, S. K. Radhakrishnan and S. Mohapatra, A study of the anisotropy associated with dipole asymmetry in heavy ion collisions, J. Phys. G40 (2013) 105108, [1203 . 3410].

[18] D. Teaney and L. Yan, Triangularity and Dipole Asymmetry in Heavy Ion Collisions, Phys. Rev. C83 (2011) 064904, [1010.1876].

[19] P. Bozek, Event-by-event viscous hydrodynamics for $C u+A$ u collisions at $\sqrt{s_{N N}}=200$ GeV, Phys. Lett. B717 (2012) 287-290, [1208 . 1887]. 